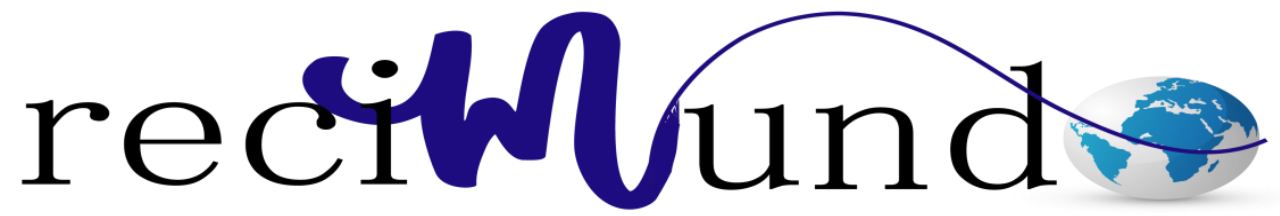

Revista Científica Mundo de la Investigación y el Conocimiento

Mercy Nathaly Alarcón Sánchez ${ }^{\text {a }}$, Liseth Pierina Pinto López ${ }^{\text {; }}$ José Benalcázar Game $^{\mathrm{c}}$

Implementar técnicas nutricionales para adultos mayores desnutridos

Revista Científica Mundo de la Investigación y el Conocimiento. Vol. 2 núm., especial, mayo, ISSN: 2588-073X, 2018, pp. 435-463

DOI: 10.26820/recimundo/2.esp.2018.435-463

Editorial Saberes del Conocimiento

Recibido: 05/12/2017 Aceptado: 15/02/2018

a. Licenciada en Dietética y Nutrición; Universidad de Guayaquil.

b. Licenciada en Dietética y Nutrición; Universidad de Guayaquil.

c. Licenciada en Dietética y Nutrición; Universidad de Guayaquil. 


\section{Implementar técnicas nutricionales para adultos mayores desnutridos}

Vol. 2, núm. Esp., (2018)

Mercy Nathaly Alarcón Sánchez; Liseth Pierina Pinto López; José Benalcázar Game

\section{RESUMEN}

La desnutrición no intencional o involuntaria del adulto mayor, contribuye a un decline progresivo en salud, afectando su estado funcional y cognitivo. La propuesta de la investigación es implantar guías alimentarias y proporcionar al personal y servicio de alimentación del asilo la formación necesaria para detectar pacientes en riesgo de desnutrición, implementar métodos de evaluación de desnutrición, sugiriendo protocolos de Tratamiento Nutricional, teniendo como objetivo general: Implementar Técnicas nutricionales para adultos mayores desnutridos. En cuanto a los objetivos específicos tenemos establecer técnicas y métodos adecuados para el adulto mayor, identificar las causas de desnutrición de los adultos mayores mediante los datos obtenidos, clasificar tipo de desnutrición e identificar la situación alimentaria y de salud de los adultos mayores, contribuir con charlas al personal de cuidado del adulto mayor para la toma de datos antropométricos y sugerir planes alimentarios adecuados para el adulto mayor. El método a utilizar fue el descriptivo de tipo de Bibliográfico documental, ya que garantiza la calidad de los fundamentos teóricos de la investigación, y de tipo Electrónico ya que se pudo conocer conceptos más actualizados para la correcta investigación del trabajo. El trabajo se realizó con pacientes mayores de 55 años del Asilo Sofía Ratinoff de Solimano en la Ciudad de Guayaquil. Se utilizaron plicómetros y cintas métricas. La antropometría fue tomada mediante circunferencia braquial, pliegue cutáneo tricipital, Talón-Rodilla, Circunferencia de la pantorrilla y Hemienvergadura. En los resultados podemos observar la mayor facilidad de toma de datos antropométricos del personal del asilo, como también en un $80 \%$ la detección de problemas nutricionales no antes visto por la falta de Nutricionista. Llegando a la conclusión de que la capacitación contínua y la actualización de datos antropométricos en el lugar, aumenta la calidad de vida de los pacientes en un $90 \%$ dejando datos necesarios para futuras investigaciones.

Palabras Clave: Técnicas nutricionales, adultos mayores, desnutrición. 


\title{
Implementar técnicas nutricionales para adultos mayores desnutridos
}

Vol. 2, núm. Esp., (2018)

Mercy Nathaly Alarcón Sánchez; Liseth Pierina Pinto López; José Benalcázar Game

\begin{abstract}
Unintentional or involuntary malnutrition in the elderly contributes to a progressive decline in health, affecting their functional and cognitive status. The research proposal is to implement food guides and provide the staff and asylum feeding service with the necessary training to detect patients at risk of malnutrition, implement malnutrition assessment methods, suggesting Nutritional Treatment protocols, with the general objective of: Implementing Techniques nutritional supplements for malnourished elderly. Regarding the specific objectives, we have to establish adequate techniques and methods for the elderly, identify the causes of malnutrition in older adults through the data obtained, classify the type of malnutrition and identify the food and health situation of older adults. talks to the care staff of the elderly adult for the collection of anthropometric data and suggest adequate food plans for the elderly. The method to be used was the descriptive type of documentary Bibliographic, since it guarantees the quality of the theoretical foundations of the research, and of Electronic type since it was possible to know more updated concepts for the correct investigation of the work. The work was carried out with patients older than 55 years of the Sofia Ratinoff Asylum of Solimano in the City of Guayaquil. Plicometers and tape measures were used. The anthropometry was taken by brachial circumference, triceps skin fold, Heel-Knee, Calf Circumference and Hemienvergadura. In the results we can observe the greater ease of taking anthropometric data of asylum personnel, as well as in $80 \%$ the detection of nutritional problems not seen before by the lack of Nutritionist. Arriving at the conclusion that the continuous training and the updating of anthropometric data in the place, increases the quality of life of the patients in a $90 \%$ leaving necessary data for future investigations
\end{abstract}

Keywords: Nutritional techniques, elderly, malnutrition. 


\section{Implementar técnicas nutricionales para adultos mayores desnutridos}

Vol. 2, núm. Esp., (2018)

Mercy Nathaly Alarcón Sánchez; Liseth Pierina Pinto López; José Benalcázar Game

\section{Introducción.}

Los cambios demográficos en la población, comienzan a requerir mayor atención de los diferentes gobiernos en el mundo. Los países industrializados comenzaron a observar estos cambios hacia la última década del siglo XX. América latina y nuestro país Ecuador, han presentado ya este comportamiento en la población, de tal suerte que en los diferentes programas asistenciales, consideran la atención de los adultos mayores. (1)

La ciudad de Guayaquil, cuenta con una tradición de años en la atención de los adultos mayores en instituciones no lucrativas conocidas como asilo, hospicios, casa de adultos etc., uno de ellos Asilo Sofia Ratinoff de Solimano, comenzó con la voluntad de dos personas visionarias preocupadas por la asistencia de aquellos que necesitaban de un lugar donde pasar sus últimos días. (2)

Las instituciones de asistencia para adultos mayores en Guayaquil cuentan con un marco legal que les da certidumbre jurídica, es verdad que se ha avanzado en infraestructura, que la atención es más integral, los profesionales de la salud voluntarios, hacen esfuerzos por mejorar la calidad de vida de los adultos mayores, pero resulta insuficiente, pues no se cuenta con la inversión sólida que garantice la cobertura de las necesidades más elementales de este grupo poblacional, como lo es la nutrición, atención médica y medicinas para la diversidad de patologías que se presentan durante el proceso de envejecimiento. (3)

La malnutrición es una enfermedad que resulta de una nutrición inadecuada, y varía de la obesidad a la desnutrición. Mientras que la obesidad está recibiendo una importante atención por parte de los profesionales sanitarios, medios y entornos políticos, la desnutrición es muchas 


\section{Implementar técnicas nutricionales para adultos mayores desnutridos}

Vol. 2, núm. Esp., (2018)

Mercy Nathaly Alarcón Sánchez; Liseth Pierina Pinto López; José Benalcázar Game

veces pasada por alto. Sin embargo, frecuentemente complica la evolución clínica en diferentes situaciones de enfermedades agudas y crónicas que afectan a múltiples órganos en niños, adultos y mayores. (4)

La desnutrición aumenta las cifras de morbilidad, mortalidad, ingresos hospitalarios y duración de la estancia. Esta desnutrición se produce entre otras cosas por déficit de nutrientes, por ingesta inadecuada, por aumento de las pérdidas o por aumento de los requerimientos de nutrientes. (5)

La prevalencia y consecuencias de la desnutrición afecta al $60 \%$ de las personas ingresadas en instituciones/residencias y al $40 \%$ de los pacientes hospitalizados y alrededor del $5 \%$ de la población general. Es frecuente, no reconocida ni tratada, pero puede ser prevenida/limitada; afecta a la recuperación de la enfermedad e incrementa la morbi-mortalidad. La desnutrición es un problema común en todos los niveles de atención sanitaria, desde atención primaria a especializada y en centros de atención geriátrica.

La propuesta de la investigación es sugerir guías y proporcionar a los cuidadores y personal del servicio de alimentación la formación en nutrición necesaria para detectar pacientes en riesgo de desnutrición y establecer las medidas pertinentes para su resolución así como implementar métodos de evaluación de la desnutrición relacionada con la enfermedad haciendo y desarrollando protocolos de Tratamiento Nutricional, estandarizar un plan de monitorización de los cuidados y tratamientos nutricionales imprescindible en la evolución del adulto mayor desnutrido. Realizar un registro de los diagnósticos de los pacientes desnutridos relacionada con la enfermedad. Sistematizar un procedimiento de evaluación de resultados en salud, considerando la calidad y los costes de las medidas establecidas. 


\section{Implementar técnicas nutricionales para adultos mayores desnutridos}

Vol. 2, núm. Esp., (2018)

Mercy Nathaly Alarcón Sánchez; Liseth Pierina Pinto López; José Benalcázar Game

En el lugar se han realizado varios trabajos en el campo de la Nutrición, el más reciente fue a cerca de la utilización de los aditivos naturales en las preparaciones de las comidas para los adultos mayores.

Los métodos que se aplicarán en la investigación son: Analítico para observar las causas, la naturaleza y los efectos del problema en el asilo y Científico por que por medio de libros, revistas científicas y referencias de internet se podrá realizar con mayor éxito el trabajo de investigación.

Se realizará un estudio transversal el cual examina la relación entre la enfermedad y una serie de variables en una población determinada y en un momento del tiempo, pueden también describir características o grados de enfermedad, también se utilizara el estudio cuantitativo que va a permitir examinar los datos de manera científica, o más específicamente en forma numérica, generalmente con ayuda de herramientas del campo de la estadística

La atención nutricional en el asilo ha de orientarse hacia la identificación de los cambios fisiológicos a lo largo del trabajo investigativo, para darles respuesta y proteger así al anciano de un declive evitable. Las comidas atractivas y sabrosas, servidas en un ambiente que anime a comer de forma autónoma, o la ayuda para comer cuando sea necesaria, para así favorecer el bienestar nutricional del adulto mayor.

Es posible realizar el presente trabajo investigativo porque se atenderá minuciosamente el estado nutricional del adulto mayor, indicando, siguiendo y modificando las inquietudes del personal de servicio de alimentación y cuidadores de los adultos mayores, cuando sea necesario, y de común acuerdo con el médico responsable del paciente, asegurando condiciones 


\section{Implementar técnicas nutricionales para adultos mayores desnutridos}

Vol. 2, núm. Esp., (2018)

Mercy Nathaly Alarcón Sánchez; Liseth Pierina Pinto López; José Benalcázar Game

adecuadas de indicación, prescripción, preparación, conservación y administración, controles clínicos y de laboratorio y el correcto seguimiento nutricional.

Se cuenta con recurso humano necesarias para la investigación, personas dotadas de características propias de personalidad y de individualidad, aspiraciones, valores, actitudes, motivaciones y objetivos individuales dispuestas a actuar en conjunto para el bien del adulto mayor y recibir la ayuda necesaria en la parte nutricional; en cuanto a los recursos económicos la institución no tiene como objetivo el lucro económico; en consecuencia, los ingresos que obtienen y que solo estén constituidos por cuotas sociales que aportan sus asociados, para el financiamiento de las actividades sociales que realiza, en cuanto a los recursos tecnológicos, lamentablemente la institución no cuenta con los suficiente, pero si con lo necesario.

La presente investigación tiene como objetivo implementar técnicas nutricionales para adultos mayores desnutridos del Asilo Sofia Ratinoff y Solimano en el período de septiembre a febrero 2013-2014.

\section{Metodología.}

\section{Diseño de la investigación}

La presente investigación, será de tipo bibliográfico documental el cual ocupa un lugar importante, ya que garantiza la calidad de los fundamentos teóricos de la investigación, ayuda a la recolección, selección, clasificación, evaluación y análisis de contenido del material impreso y gráfico, físico y/o virtual que servirá de fuente teórica, conceptual y/o metodológica para la investigación científica determinada y de tipo electrónico ya que se pudo conocer conceptos más actualizados para la correcta investigación del trabajo 


\section{Implementar técnicas nutricionales para adultos mayores desnutridos}

Vol. 2, núm. Esp., (2018)

Mercy Nathaly Alarcón Sánchez; Liseth Pierina Pinto López; José Benalcázar Game

\section{Tipo de estudio}

Para tener un mayor alcance y conocimiento acerca del tema, el estudio se dividió en dos tipos: estudio transversal y estudio cuantitativo.

El estudio transversal el cual examina la relación entre la enfermedad y una serie de variables en una población determinada y en un momento del tiempo, pueden también describir características o grados de enfermedad, y el estudio cuantitativo que va a permitir examinar los datos de manera científica, o más específicamente en forma numérica, generalmente con ayuda de herramientas del campo de la estadística.

\section{Nivel de estudio}

Se utilizó el nivel de estudio aplicada ya que esta confronta la teoría con la realidad y los métodos que se utilizaron fueron transversal ya que mide a la vez la prevalencia de la exposición y del efecto en la muestra en un solo momento temporal; es decir, permite estimar la magnitud y distribución de la enfermedad en un momento dado; y cuantitativo porque se estudió a cada uno de los pacientes.

\section{Población}

En dicha investigación se tomaron a pacientes con desnutrición que están en el Asilo Sofia Ratinoff de Solimano, la cantidad de pacientes que consta es de 180. 


\section{Implementar técnicas nutricionales para adultos mayores desnutridos}

Vol. 2, núm. Esp., (2018)

Mercy Nathaly Alarcón Sánchez; Liseth Pierina Pinto López; José Benalcázar Game

\begin{tabular}{|l|c|}
\hline Población & Cantidad \\
\hline Mujeres & 85 \\
\hline Hombres & 95 \\
\hline TOTAL & $\mathbf{1 8 0}$ \\
\hline
\end{tabular}

Fuente: Asilo Sofia Ratinoff de Solimano

Autora: Mercy Alarcón Sánchez

Muestra

La muestra recolectó pacientes mayores de 55 años con desnutrición que residen en el Asilo Sofía Rattinoff de Solimano localizado en la Ciudad de Guayaquil, esta evaluación nutricional se la realizara durante un intervalo de seis meses, en la cual contaremos con la ayuda del Personal de Servicio de Alimentación, Licenciados en Enfermería y Auxiliares de Enfermería.

\begin{tabular}{|l|c|}
\hline Muestra & Cantidad \\
\hline Mujeres & 44 \\
\hline Hombres & 57 \\
\hline TOTAL & $\mathbf{9 7}$ \\
\hline
\end{tabular}

Recolección de la información

Se estableció mediante todas las mediciones antropométricas las cuales fueron tomadas mediante técnicas estándar e incluirán: circunferencia media del brazo, pliegue cutáneo tricipital, longitud talón-rodilla, circunferencia media de la pierna, hemienvergadura, peso y talla, esta se la realizara una vez por mes para analizar la evolución del paciente. 


\section{Implementar técnicas nutricionales para adultos mayores desnutridos}

Vol. 2, núm. Esp., (2018)

Mercy Nathaly Alarcón Sánchez; Liseth Pierina Pinto López; José Benalcázar Game

Se realizarán charlas Nutricionales al Personal de Servicio de Alimentación con el fin de mejorar la alimentación del Asilo, así mismo se dará la respectiva explicación a los Licenciados en Enfermería y Auxiliares de Enfermería sobre la antropometría para que estos una vez culminado el trabajo de investigación sepan cómo coger los datos antropométricos de los Adultos Mayores

Los resultados recogidos se los analizarán por medio de fórmulas, y tablas realizadas en Excel.

El objetivo de los resultados obtenidos es para poder realizar sugerencias dirigidas a los directivos del asilo, cuidadores y personal de servicio de alimentación encargados de los Adultos Mayores.

Instrumentos de la investigación

Los materiales a utilizar tenemos: Plicómetros, Cinta Métrica, Historia Clínica, Tablas Excel, una entrevista estructurada a cada paciente que consistió en la recolección de datos sobre la condición socioeconómica y la respectiva Valoración Antropométrica teniendo en cuenta que el lugar donde se realizara la investigación no consta con el equipamiento necesario para poder realizar la evaluación nutricional en los Adultos Mayores. 


\section{Implementar técnicas nutricionales para adultos mayores desnutridos}

Vol. 2, núm. Esp., (2018)

Mercy Nathaly Alarcón Sánchez; Liseth Pierina Pinto López; José Benalcázar Game

\section{Resultados.}

Pregunta No.1.- Clasificación según el Género como parámetro nutricional, para ver los niveles de Desnutrición en Pacientes Adultos Mayores.

Cuadro $\mathbf{N}^{\circ} 1$

\begin{tabular}{|c|c|c|c|}
\hline Ítem & Género & Frecuencia & \% \\
\hline 1 & Femenino & 44 & 45 \\
\hline 2 & Masculino & 53 & 55 \\
\hline & TOTAL & 97 & 100 \\
\hline
\end{tabular}

Fuente: Asilo Sofia Ratinoff de Solimano

Autora: Mercy Alarcón Sánchez

Gráfico No, 1

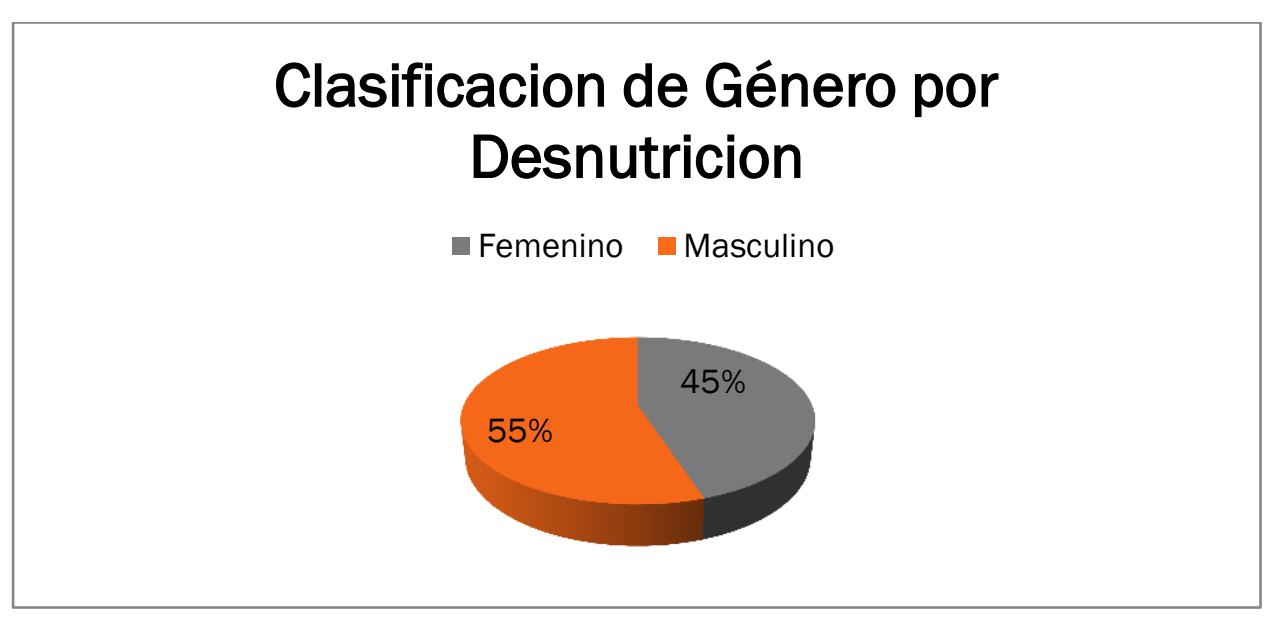

Fuente: Asilo Sofia Ratinoff de Solimano

Autora: Mercy Alarcón Sánchez 


\section{Implementar técnicas nutricionales para adultos mayores desnutridos}

Vol. 2, núm. Esp., (2018)

Mercy Nathaly Alarcón Sánchez; Liseth Pierina Pinto López; José Benalcázar Game

\section{Análisis.-}

En los pacientes adultos mayores desnutridos la mayor incidencia de género, refleja los siguientes rangos: la desnutrición predomina en el sexo masculino con un 55\% y no muy lejos se encuentra el sexo femenino con un $45 \%$.

Pregunta No.2.- Clasificación según la Edad como parámetro nutricional en Pacientes Adultos Mayores.

Cuadro $\mathbf{N}^{\circ} 2$

\begin{tabular}{|l|l|l|l|}
\hline Ítem & Edad & Frecuencia & \% \\
\hline 1 & $55-65$ & 16 & 16 \\
\hline 2 & $66-75$ & 26 & 27 \\
\hline 3 & $76-85$ & 30 & 31 \\
\hline 4 & $86-95$ & 25 & 26 \\
\hline & TOTAL & 97 & 100 \\
\hline
\end{tabular}

Fuente: Asilo Sofia Ratinoff de Solimano

Autora: Mercy Alarcón Sánchez 


\section{Implementar técnicas nutricionales para adultos mayores desnutridos}

Vol. 2, núm. Esp., (2018)

Mercy Nathaly Alarcón Sánchez; Liseth Pierina Pinto López; José Benalcázar Game

\section{Gráfico No, 2}

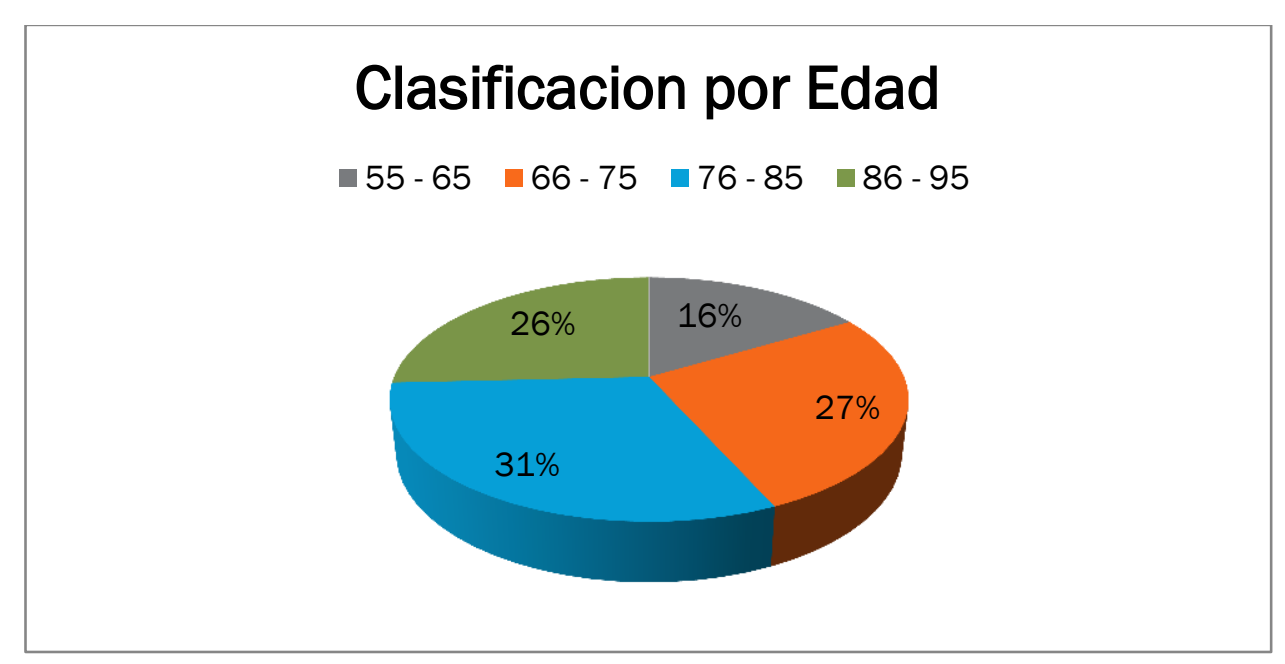

Fuente: Asilo Sofia Ratinoff de Solimano

Autora: Mercy Alarcón Sánchez

\section{Análisis.-}

Aquí podemos observar la Clasificación de los adultos mayores desnutridos según su edad en donde se refleja con un porcentaje del $31 \%$ las edades que predominan son de $76-85$ años, luego con un $27 \%$ las edades de entre 66-75, muy seguido un porcentaje del $26 \%$ las edades de $86-95$ años y del $16 \%$ en donde tenemos las edades de 55-65. 


\section{Implementar técnicas nutricionales para adultos mayores desnutridos}

Vol. 2, núm. Esp., (2018)

Mercy Nathaly Alarcón Sánchez; Liseth Pierina Pinto López; José Benalcázar Game

Pregunta No.3.- Clasificación según el Peso en Kilogramos como parámetro.

\section{Cuadro $\mathbf{N}^{\circ} 3$}

\begin{tabular}{|l|l|l|l|}
\hline Ítem & Peso & Frecuencia & \% \\
\hline 1 & $10-25$ & 34 & 35 \\
\hline 2 & $26-35$ & 34 & 35 \\
\hline 3 & $36-45$ & 22 & 23 \\
\hline 4 & $46-55$ & 6 & 6 \\
\hline 5 & $56-70$ & 1 & 1 \\
\hline & TOTAL & 97 & 100 \\
\hline
\end{tabular}

Fuente: Asilo Sofia Ratinoff de Solimano

Autora: Mercy Alarcón Sánchez

Gráfico No, 3

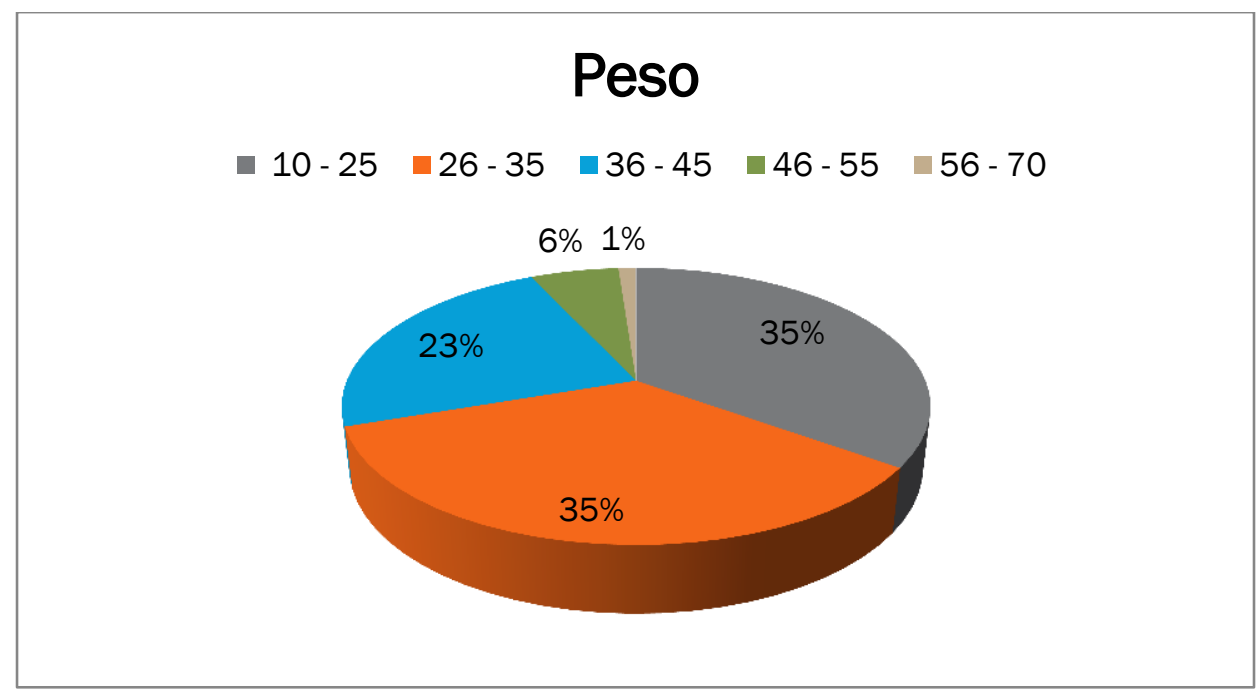

Fuente: Asilo Sofia Ratinoff de Solimano

Autora: Mercy Alarcón Sánchez 


\section{Implementar técnicas nutricionales para adultos mayores desnutridos}

Vol. 2, núm. Esp., (2018)

Mercy Nathaly Alarcón Sánchez; Liseth Pierina Pinto López; José Benalcázar Game

\section{Análisis.-}

En este cuadro podemos observar los pesos de los adultos mayores en kilogramos, los cuales reflejan porcentajes iguales del $35 \%$ con pesos de $10-25 \mathrm{~kg}$ junto con $26-35 \mathrm{~kg}$, seguido de un $23 \%$ los pesos de $36-45 \mathrm{~kg}$, y bajos porcentajes como el $6 \%$ los pesos de $46-55 \mathrm{~kg}$ y $1 \%$ de 56 $70 \mathrm{~kg}$.

Pregunta No.4.- Clasificación por Índice de Masa Corporal como parámetro, para determinar el estado Nutricional en Pacientes Adultos Mayores.

\section{Cuadro $\mathbf{N}^{\circ} 4$}

\begin{tabular}{|l|l|l|l|l|}
\hline Ítem & $\begin{array}{l}\text { Índice de Masa } \\
\text { Corporal }\end{array}$ & Rangos & Frecuencia & $\%$ \\
\hline 1 & Desnutrición & $16-16,99$ & 69 & 71 \\
\hline 2 & Bajo Peso & $17-18,40$ & 4 & 4 \\
\hline 3 & Normopeso & $18-24$ & 22 & 23 \\
\hline 4 & Sobrepeso & 25 & 2 & 2 \\
\hline & TOTAL & - & 97 & 100 \\
\hline
\end{tabular}

Fuente: Asilo Sofia Ratinoff de Solimano

Autora: Mercy Alarcón Sánchez 


\section{Implementar técnicas nutricionales para adultos mayores desnutridos}

Vol. 2, núm. Esp., (2018)

Mercy Nathaly Alarcón Sánchez; Liseth Pierina Pinto López; José Benalcázar Game

Gráfico No, 4

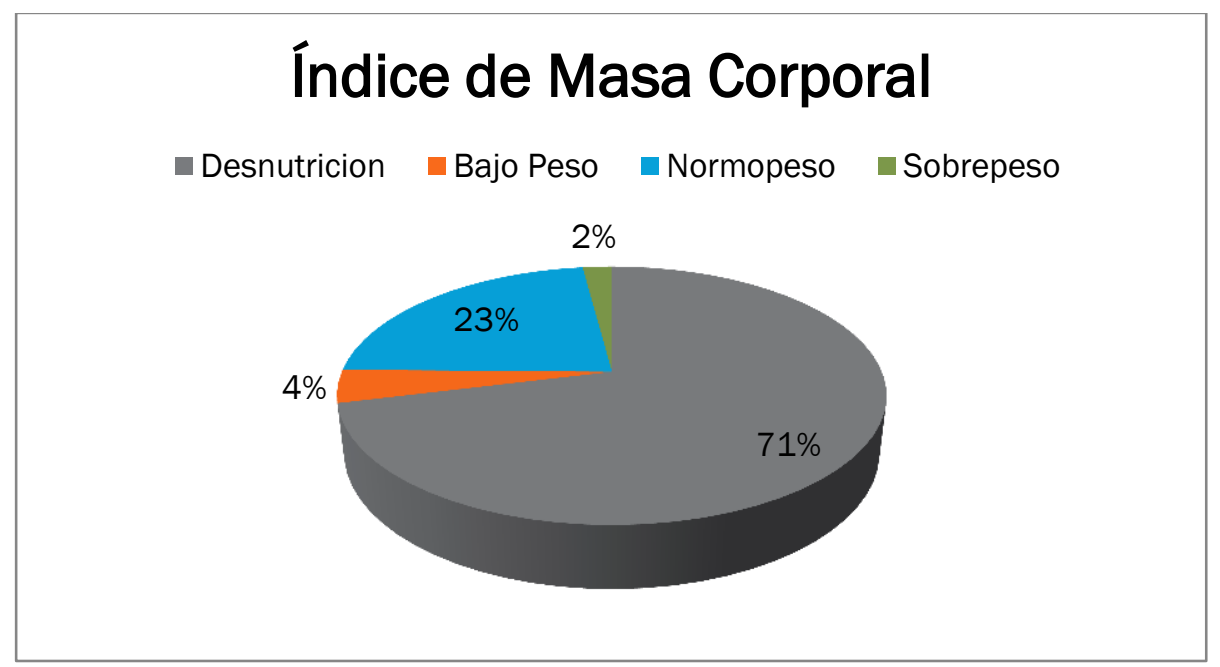

Fuente: Asilo Sofia Ratinoff de Solimano

Autora: Mercy Alarcón Sánchez

\section{Análisis.-}

Este cuadro nos refleja el IMC de los adultos mayores, en donde se refleja los siguientes rangos:

Desnutrición 71\%, Normopeso 23\%, Bajo Peso 4\%, y Sobrepeso 2\%. 


\section{Implementar técnicas nutricionales para adultos mayores desnutridos}

Vol. 2, núm. Esp., (2018)

Mercy Nathaly Alarcón Sánchez; Liseth Pierina Pinto López; José Benalcázar Game

Pregunta No.5.- Pliegue Cutáneo Tricipital como parámetro nutricional, para evaluar la grasa subcutánea en Pacientes Adultos Mayores Desnutridos.

\section{Cuadro $\mathbf{N}^{0} 5$}

\begin{tabular}{|l|l|l|l|l|}
\hline Ítem & $\begin{array}{l}\text { Pliegue Cutáneo } \\
\text { Tricipital }\end{array}$ & Rangos & Frecuencia & $\%$ \\
\hline 1 & Exceso de grasa & $\geq 110$ & 16 & 17 \\
\hline 2 & Normal & $90-100$ & 24 & 25 \\
\hline 3 & Deficiencia Leve & $51-89$ & 48 & 49 \\
\hline 4 & Deficiencia Modera & $30-50$ & 8 & 8 \\
\hline 5 & Deficiencia Severa & $<30$ & 1 & 1 \\
\hline & TOTAL & - & 97 & 100 \\
\hline
\end{tabular}

Fuente: Asilo Sofia Ratinoff de Solimano

Autora: Mercy Alarcón Sánchez 


\section{Implementar técnicas nutricionales para adultos mayores desnutridos}

Vol. 2, núm. Esp., (2018)

Mercy Nathaly Alarcón Sánchez; Liseth Pierina Pinto López; José Benalcázar Game

\section{Gráfico No, 5}

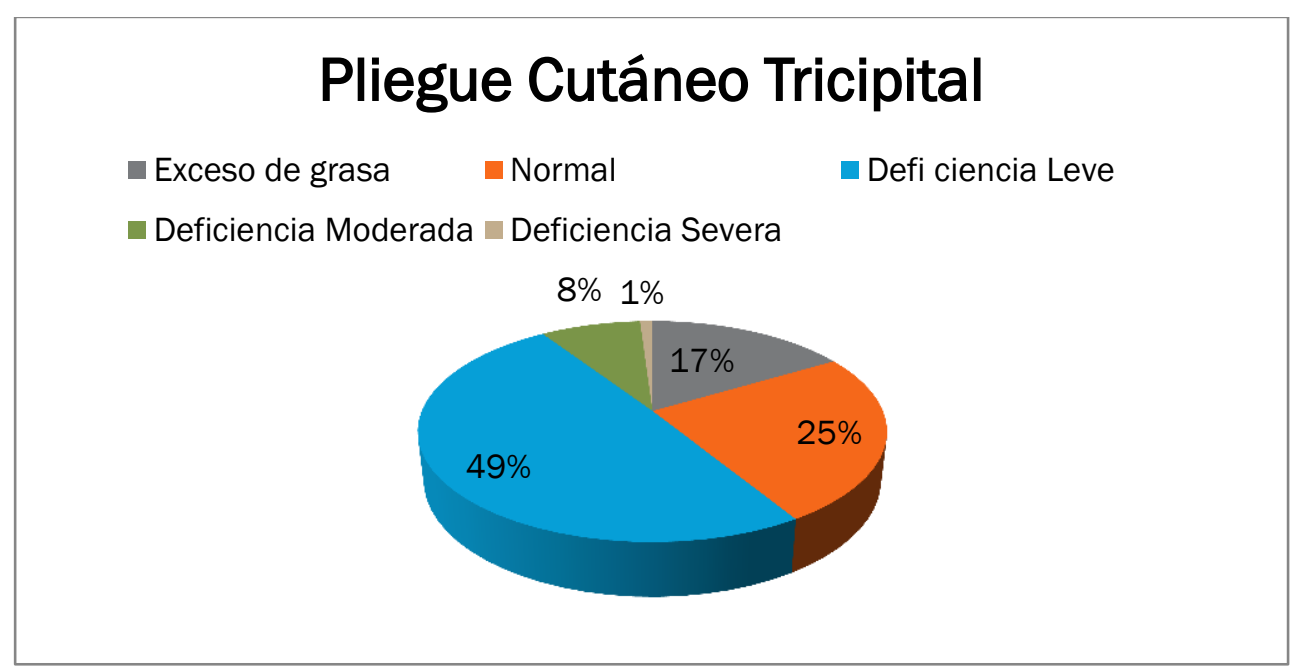

Fuente: Asilo Sofia Ratinoff de Solimano

Autora: Mercy Alarcón Sánchez

\section{Análisis.-}

En los pacientes adultos mayores desnutridos, el Pliegue Cutáneo Tricipital los cuales reflejan porcentajes del 49\% con Deficiencia Leve, con el 25\% Normal, Exceso de Grasa 17\%, Deficiencia Moderada $8 \%$ y Deficiencia Severa $1 \%$ 


\section{Implementar técnicas nutricionales para adultos mayores desnutridos}

Vol. 2, núm. Esp., (2018)

Mercy Nathaly Alarcón Sánchez; Liseth Pierina Pinto López; José Benalcázar Game

Pregunta No.6.- Circunferencia Media del Brazo como parámetro nutricional, para evaluar la grasa subcutánea en Pacientes Adultos Mayores Desnutridos.

\section{Cuadro $\mathbf{N}^{0} 6$}

\begin{tabular}{|c|l|l|l|l|}
\hline Ítem & $\begin{array}{l}\text { Circunferencia Media } \\
\text { del Brazo }\end{array}$ & Rangos & Frecuencia & $\%$ \\
\hline 1 & Obesidad & $\geq 120$ & - & - \\
\hline 2 & Exceso de Peso & $120-110$ & - & - \\
\hline 3 & Normal & $90-110$ & - & - \\
\hline 4 & Desgaste Leve & $81-90$ & - & - \\
\hline 5 & Desgaste Moderado & $70-80$ & - & - \\
\hline 6 & Desgaste Severo & $<70$ & 97 & 100 \\
\hline & TOTAL & - & 97 & 100 \\
\hline
\end{tabular}

Fuente: Asilo Sofia Ratinoff de Solimano

Autora: Mercy Alarcón Sánchez 


\section{Implementar técnicas nutricionales para adultos mayores desnutridos}

Vol. 2, núm. Esp., (2018)

Mercy Nathaly Alarcón Sánchez; Liseth Pierina Pinto López; José Benalcázar Game

\section{Gráfico No, 6}

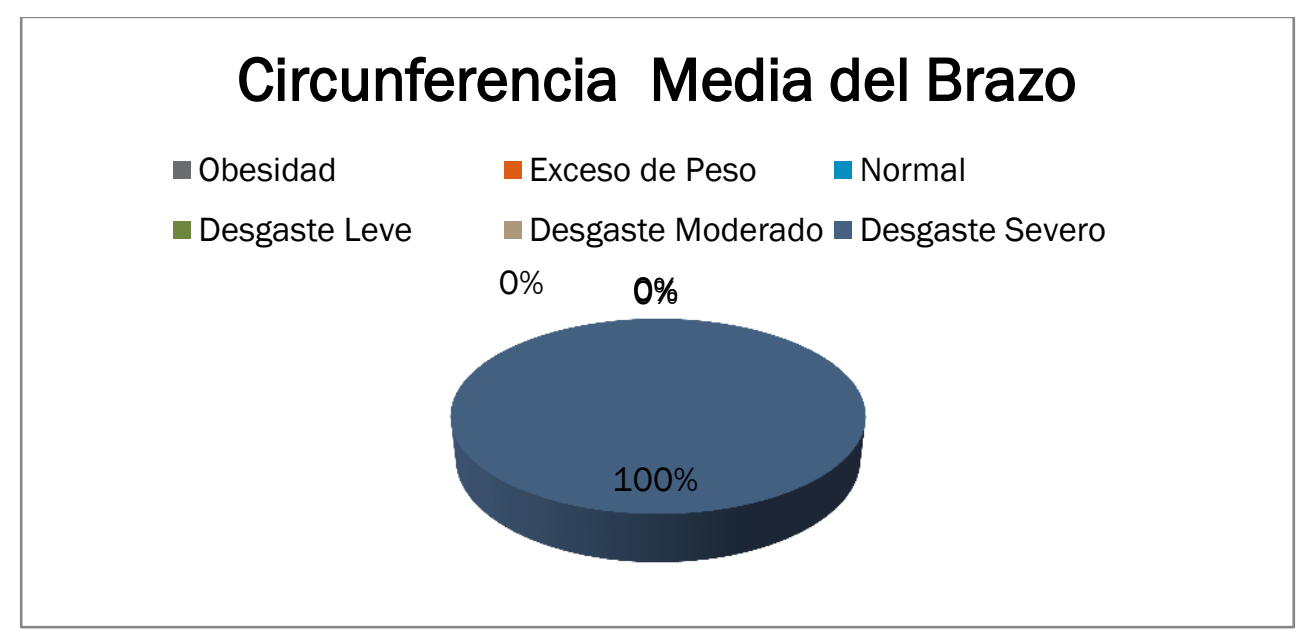

Fuente: Asilo Sofia Ratinoff de Solimano

Autora: Mercy Alarcón Sánchez

\section{Análisis.-}

En los pacientes adultos mayores desnutridos, la Circunferencia Media del Brazo refleja en los Pacientes Adultos Mayores Desnutridos refleja que todos estos pacientes tienen un 100\% de Desgaste Severo. 


\section{Implementar técnicas nutricionales para adultos mayores desnutridos}

Vol. 2, núm. Esp., (2018)

Mercy Nathaly Alarcón Sánchez; Liseth Pierina Pinto López; José Benalcázar Game

Pregunta No.7.- Clasificación de los Tipos de Desnutrición como parámetro nutricional en Pacientes Adultos Mayores Desnutridos

\section{Cuadro $\mathbf{N}^{\circ} 7$}

\begin{tabular}{|l|l|l|l|l|}
\hline Ítem & $\begin{array}{l}\text { Tipos de } \\
\text { Desnutrición }\end{array}$ & Rangos & Frecuencia & $\%$ \\
\hline 1 & Desnutrición Severa & $<16$ & 82 & 85 \\
\hline 2 & $\begin{array}{l}\text { Desnutrición } \\
\text { Moderada }\end{array}$ & $16-16,99$ & 8 & 8 \\
\hline 3 & Desnutrición Leve & $17-18,40$ & 7 & 7 \\
\hline & TOTAL & - & 97 & 100 \\
\hline
\end{tabular}

Fuente: Asilo Sofia Ratinoff de Solimano

Autora: Mercy Alarcón Sánchez 


\section{Implementar técnicas nutricionales para adultos mayores desnutridos}

Vol. 2, núm. Esp., (2018)

Mercy Nathaly Alarcón Sánchez; Liseth Pierina Pinto López; José Benalcázar Game

\section{Gráfico No, 7}

\section{Tipos de Desnutrición}

-Desnutricion Severa $\square$ Desnutricion Moderada $\square$ Desnutricion Leve

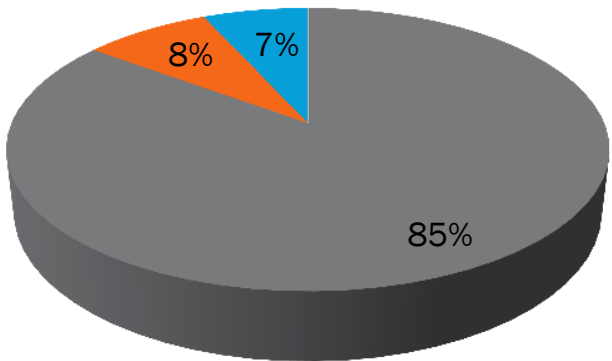

Fuente: Asilo Sofia Ratinoff de Solimano

Autora: Mercy Alarcón Sánchez

\section{Análisis.-}

En este cuadro se puede observar que hay un mayor porcentaje de Desnutrición Severa con un 85\%, y con un mínimo porcentaje del 8\% en Desnutrición Moderada y un 7\% Desnutrición Leve. 


\section{Implementar técnicas nutricionales para adultos mayores desnutridos}

Vol. 2, núm. Esp., (2018)

Mercy Nathaly Alarcón Sánchez; Liseth Pierina Pinto López; José Benalcázar Game

Pregunta No.8.- Enfermedades Neurológicas como parámetro nutricional, para ver los niveles de Desnutrición en Pacientes Adultos Mayores.

\section{Cuadro $\mathbf{N}^{\circ} 8$}

\begin{tabular}{|l|l|l|l|}
\hline Ítem & Enfermedades Neurológicas & Frecuencia & $\%$ \\
\hline 1 & Alzheimer & 35 & 36 \\
\hline 2 & Parkinson & 40 & 41 \\
\hline 3 & Epilepsia & 22 & 23 \\
\hline & TOTAL & 97 & 100 \\
\hline
\end{tabular}

Fuente: Asilo Sofia Ratinoff de Solimano

Autora: Mercy Alarcón Sánchez

\section{Gráfico No, 8}

\section{Enfermedades Neurologicas}

घ Alzheimer $\quad$ Parkinson Epilepsia

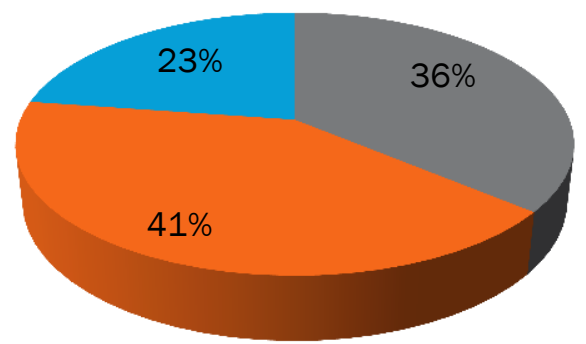

Fuente: Asilo Sofia Ratinoff de Solimano

Autora: Mercy Alarcón Sánchez 


\section{Implementar técnicas nutricionales para adultos mayores desnutridos}

Vol. 2, núm. Esp., (2018)

Mercy Nathaly Alarcón Sánchez; Liseth Pierina Pinto López; José Benalcázar Game

\section{Análisis.-}

En los pacientes adultos mayores desnutridos la mayor incidencia de Enfermedades Neurológicas refleja que hay un mayor porcentaje de Parkinson con un $41 \%$, con un $36 \%$ de Alzheimer y un $23 \%$ en Epilepsia.

Pregunta No.9.- Clasificación según Áreas como parámetro nutricional, para ver los niveles de Desnutrición en áreas indigentes y pagadas en Pacientes Adultos Mayores.

\section{Cuadro $\mathbf{N}^{\circ} 9$}

\begin{tabular}{|l|l|l|l|}
\hline Ítem & Áreas & Frecuencia & $\%$ \\
\hline 1 & Indigentes & 51 & 52 \\
\hline 2 & Pagada & 46 & 48 \\
\hline & TOTAL & 97 & 100 \\
\hline
\end{tabular}

Fuente: Asilo Sofia Ratinoff de Solimano

Autora: Mercy Alarcón Sánchez 


\section{Implementar técnicas nutricionales para adultos mayores desnutridos}

Vol. 2, núm. Esp., (2018)

Mercy Nathaly Alarcón Sánchez; Liseth Pierina Pinto López; José Benalcázar Game

\section{Gráfico No, 9}

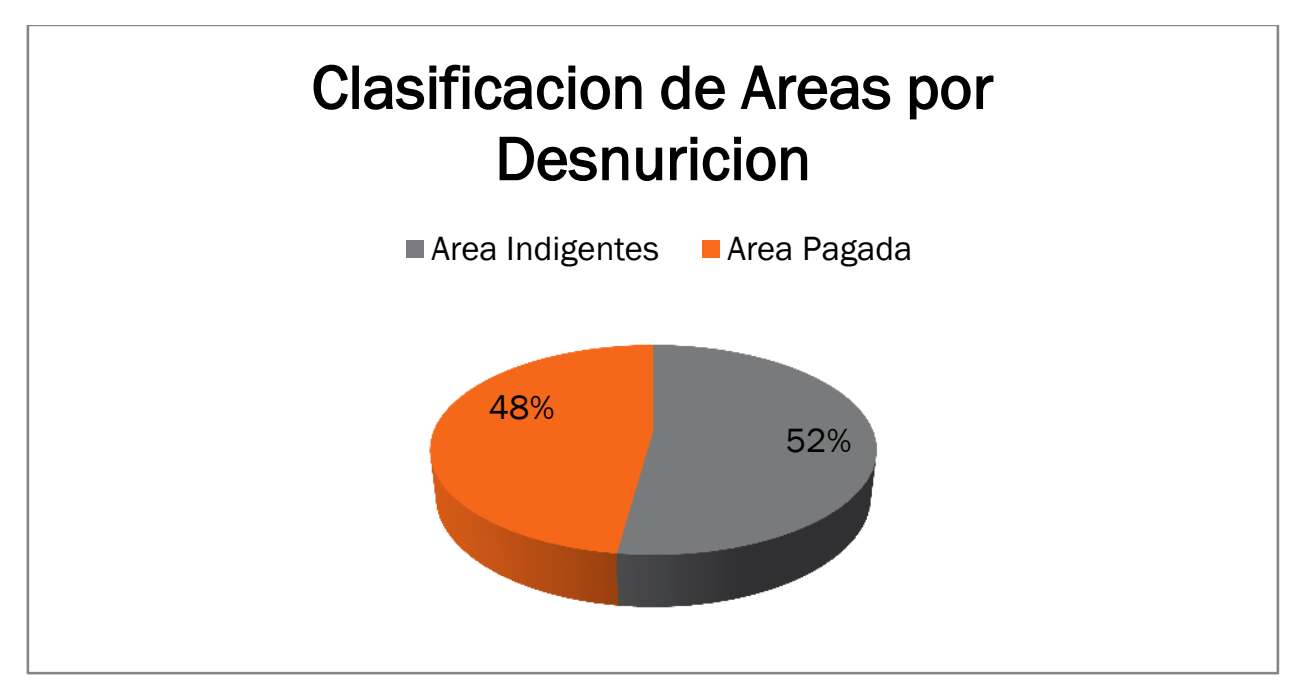

Fuente: Asilo Sofia Ratinoff de Solimano

Autora: Mercy Alarcón Sánchez

\section{Análisis.-}

En el cuadro identifica los pacientes adultos mayores en donde refleja que el área con mayor desnutrición es el Área de Indigentes con el 52\% y con un 48\% en el Área Pagada 


\section{Implementar técnicas nutricionales para adultos mayores desnutridos}

Vol. 2, núm. Esp., (2018)

Mercy Nathaly Alarcón Sánchez; Liseth Pierina Pinto López; José Benalcázar Game

Pregunta No.10.- Clasificación según el IMC de Entrada con el de Salida como parámetro nutricional, para ver los niveles de Desnutrición en Pacientes Adultos Mayores.

\section{Cuadro $\mathbf{N}^{\mathbf{1}} 10$}

\begin{tabular}{|l|l|l|l|}
\hline Ítem & IMC & Frecuencia & $\%$ \\
\hline 1 & Entrada & 60 & 63 \\
\hline 2 & Salida & 37 & 37 \\
\hline & TOTAL & 97 & 100 \\
\hline
\end{tabular}

Fuente: Asilo Sofia Ratinoff de Solimano

Autora: Mercy Alarcón Sánchez 


\section{Implementar técnicas nutricionales para adultos mayores desnutridos}

Vol. 2, núm. Esp., (2018)

Mercy Nathaly Alarcón Sánchez; Liseth Pierina Pinto López; José Benalcázar Game

\section{Gráfico No, 10}

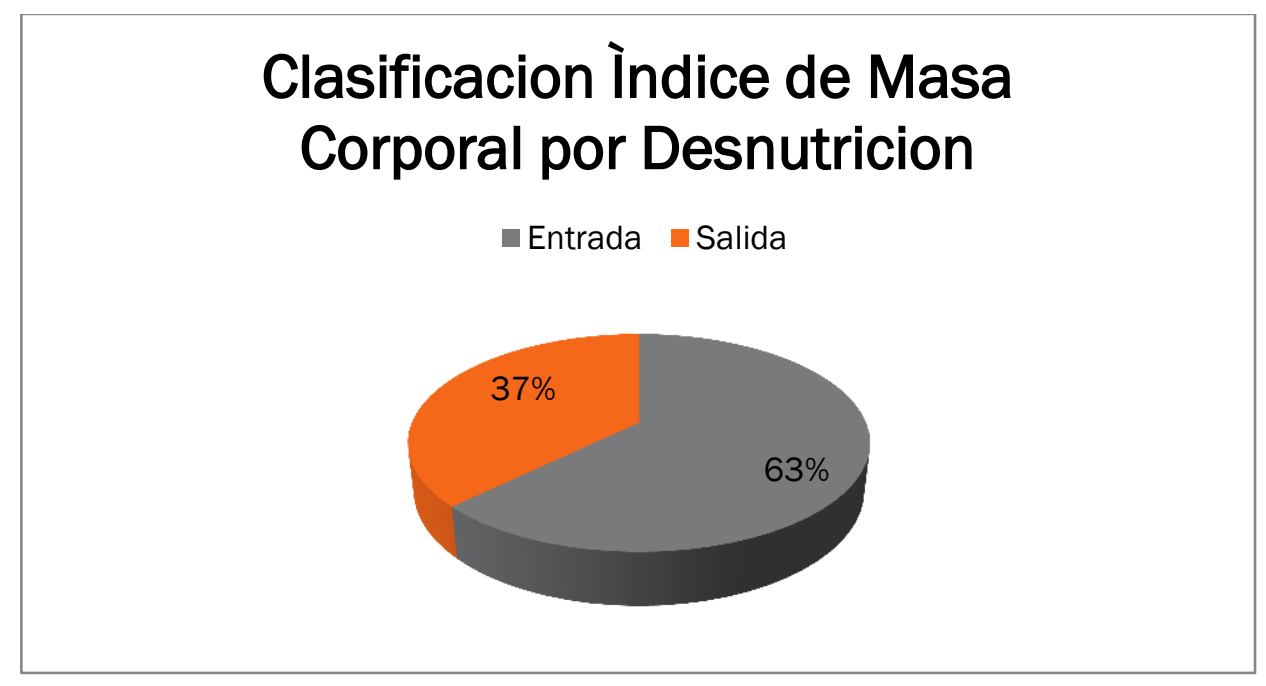

Fuente: Asilo Sofia Ratinoff de Solimano

Autora: Mercy Alarcón Sánchez

\section{Análisis.-}

En los pacientes adultos mayores desnutridos, en la toma de antropometría al inicio del trabajo tienen un porcentaje del $63 \%$ y al final del trabajo con un porcentaje del $37 \%$.

\section{Conclusiones.}

La nutrición humana en el campo de la medicina precisa de un conocimiento interdisciplinario que ayude a la prevención y tratamiento de la enfermedad, lo que implica a numerosos profesionales de la salud, en particular, para prevenir y/o tratar la malnutrición del 


\section{Implementar técnicas nutricionales para adultos mayores desnutridos}

Vol. 2, núm. Esp., (2018)

Mercy Nathaly Alarcón Sánchez; Liseth Pierina Pinto López; José Benalcázar Game

paciente, por lo cual es importante la existencia de un equipo multidisciplinar que integre el diagnóstico y tratamiento de la desnutrición en el adulto mayor.

La evaluación periódica de la nutrición en los adultos mayores puede ayudar a retrasar el inicio o gravedad de enfermedades crónicas.

- $\quad$ Existe un $63 \%$ de adultos mayores desnutridos a los cuales no se les realiza una valoración antropométrica y/o nutricional.

- Los adultos mayores no llevan una alimentación de acuerdo a la edad y/o enfermedades propias del longevo.

- La alimentación de los adultos mayores no es la correcta en cuanto a nutrientes.

- Algunos adultos mayores sufren de pérdida de apetito o poco sentido del gusto lo cual dificulta la ingesta de alimentos

- El asilo no posee los ingresos suficientes para el abastecimiento de los alimentos necesarios.

- Los adultos mayores no realizan ningún tipoi de actividad física.

\section{Bibliografía.}

1. Alvarado-García A, Lamprea-Reyes L, Murcia-Tabares K. La nutrición en el adulto mayor: una oportunidad para el cuidado de enfermería. Enfermería universitaria. 2017 Sep; 14(3): p. 199-206.

2. Organización Mundial de la Salud. Informe mundial sobre el envejecimiento y la salud Ginebra: Informe de un Grupo Científico de la OMS; 2015.

3. Varela-Pinedo L. Nutrición en el adulto mayor: Nutrition in the elder population. Rev Med Hered. 2013 Oct; 14(13): p. 184-189.

4. Restrepo-M S, Morales-G R, Ramírez-G M. Los hábitos alimentarios en el adulto mayor y su relación con los procesos protectores y deteriorantes en salud. Rev Chil Nutr. 2006; 33(1): p. 


\section{Implementar técnicas nutricionales para adultos mayores desnutridos}

Vol. 2, núm. Esp., (2018)

Mercy Nathaly Alarcón Sánchez; Liseth Pierina Pinto López; José Benalcázar Game

$500-510$.

5. García-Talavera N, Martín-Pozuelo G. Detección del riesgo de desnutrición en ancianos no institucionalizados. Nutr. Hosp. 2006 Feb; 21(6): p. 100-110. 